\title{
Active Liquid Crystal Tuning of Metallic Nanoantenna Enhanced Light Emission from Colloidal Quantum Dots
}

\author{
Aimi Abass, ${ }^{\dagger}$ Said Rahimzadeh-Kalaleh Rodriguez, ${ }^{\ddagger}$ Thomas Ako, ${ }^{\S}$ Tangi Aubert, ${ }^{\|, \perp}$ Marc Verschuuren, ${ }^{\#}$ \\ Dries Van Thourhout, ${ }^{\nabla, \perp}$ Jeroen Beeckman, ${ }^{\S, \perp}$ Zeger Hens, ${ }^{\|, \perp}$ Jaime Gómez Rivas, \\ and Bjorn Maes*, ${ }^{\prime}, \nabla$ \\ ${ }^{\dagger}$ Solar Cells Group, Department of Electronic and Information Systems (ELIS), Ghent University, Sint-Pietersnieuwstraat 41, B-9000 \\ Ghent, Belgium \\ ${ }^{\ddagger}$ Center for Nanophotonics, FOM Institute AMOLF, c/o Philips Research Laboratories, High Tech Campus 4, 5656 AE Eindhoven, \\ The Netherlands \\ ${ }^{\S}$ Liquid Crystals and Photonics Group, Department of Electronic and Information Systems (ELIS), Ghent University, \\ Sint-Pietersnieuwstraat 41, B-9000 Ghent, Belgium \\ "Physics and Chemistry of Nanostructure, Department of Inorganic and Physical Chemistry, Ghent University, Krijgslaan 281, \\ B-9000 Ghent, Belgium \\ ${ }^{\perp}$ Center for Nano- and Biophotonics (NB-Photonics), Ghent University, B-9000 Ghent, Belgium \\ \#Philips Research Laboratories, High Tech Campus 4, 5656 AE Eindhoven, The Netherlands \\ ${ }^{\nabla}$ Photonics Research Group (INTEC), Ghent University-IMEC, Sint-Pietersnieuwstraat 41, B-9000 Ghent, Belgium \\ - COBRA Research Institute, Eindhoven University of Technology, P.O. Box 513, 5600 MB Eindhoven, The Netherlands \\ IIMicro- and Nanophotonic Materials Group, Faculty of Science, University of Mons, Place du Parc 20, B-7000 Mons, Belgium
}

\section{Supporting Information}

ABSTRACT: A system comprising an aluminum nanoantenna array on top of a luminescent colloidal quantum dot waveguide and covered by a thermotropic liquid crystal (LC) is introduced. By heating the LC above its critical temperature, we demonstrate that the concomitant refractive index change modifies the hybrid plasmonic-photonic resonances in the system. This enables active control of the spectrum and directionality of the narrow-band $(\sim 6 \mathrm{~nm})$ enhancement of quantum dot photoluminescence by the metallic nanoantennas.

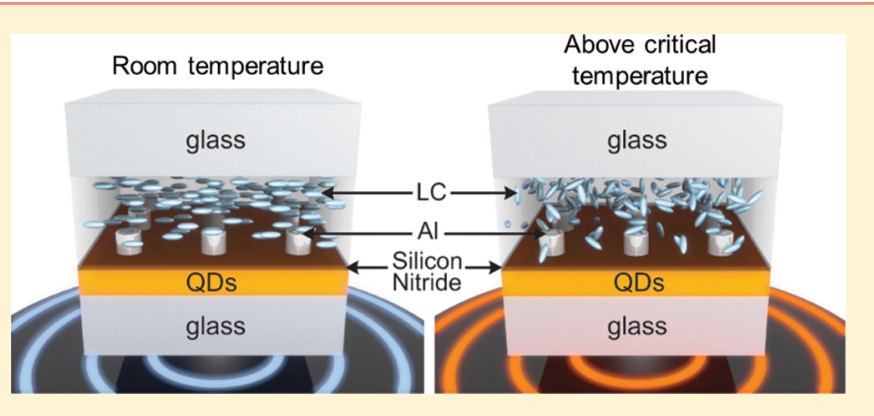

KEYWORDS: Waveguide, nanoantenna, liquid crystal, quantum dot, fluorescence

M etallic nanostructures display remarkable optical properties associated with collective excitations of conduction electrons known as localized surface plasmon resonances (LSPRs). LSPRs convert free-space radiation into localized energy and vice versa. Therefore, the term optical antenna, or nanoantenna, has emerged to describe metallic nanostructures with LSPRs coupled to light sources or receivers. ${ }^{1}$ Metallic nanoantennas can provide directivity gain, ${ }^{2-6}$ polarization control, ${ }^{7,8}$ intensity enhancements, ${ }^{9,10}$ decay rate enhancements, ${ }^{11}$ and spectral shaping. ${ }^{12}$ A long-standing goal in nanophotonics is to actively control these coupling-enhanced emission properties by means of an external tuning parameter. This can be achieved by incorporating materials with optical or geometrical properties that depend on an applied voltage, heat, strain, or illumination profile. ${ }^{13-18}$ Liquid crystals (LCs) are interesting materials for this purpose, because their tunable orientation can modify the resonance conditions of nearby optical resonators. Indeed, LCs have enabled active control of resonances in diverse metallic structures, including LSPR-based nanoantennas and propagating surface plasmon polaritons in continuous metallic films. ${ }^{19-28}$ While powerful, these approaches suffer from inherent drawbacks in the context of light emission. On one hand, the influence of LSPRs is spatially restricted to emitters located within their characteristic decay lengths (typically $<40 \mathrm{~nm}$ ). ${ }^{29}$ On the other hand, the large amount of nonradiative decay channels in continuous metallic films can lead to emission quenching. ${ }^{30}$ An interesting approach to overcome these limitations consists of coupling LSPRs in individual nanoantennas to long-range photonic modes in dielectric structures. Such coupling occurs, for example, in periodic arrays of metallic nanostructures. LSPRs in individual 
nanoantennas can couple to diffracted ${ }^{31-33}$ or guided modes, ${ }^{34,35}$ resulting in hybrid plasmonic-photonic modes. The dispersion, line width, and field confinement of these hybrid modes can be designed via the geometry and dimensions of the structures. ${ }^{36,37}$ Moreover, their fields can be constructed to spatially overlap with nearby emitters extended over large areas in a polarization-, frequency- and angle-dependent manner. ${ }^{38-42}$ The long-range character of these hybrid modes is well-suited to modify the emission from spatially extended sources in the periodicity plane, while preserving subwavelength confinement out of the same plane. Thus, we envisage that active control of these hybrid modes holds great promise for applications in solid state lighting, lasers, and on-chip photon sources.

Here we demonstrate active LC tuning of a spectrally narrow photoluminescence enhancement (PLE) by a periodic array of metallic nanoantennas coupled to a waveguide. The structure we investigate consists of an aluminum nanodisk array fabricated on top of an emitting layer (acting as a waveguide) of colloidal quantum dots (QDs) and coated with a thermotropic LC. Colloidal QDs constitute ideal emitters for this purpose as they offer sharp and tunable emission properties, even at ambient or higher temperatures, in combination with a versatile processability. ${ }^{43}$ The active tuning is achieved by changing the temperature of the sample. Above a critical temperature $T_{\mathcal{c}}$, the orientation of the LC becomes randomized, and the effective refractive index switches from birefringent to isotropic. We evidence the impact of the LC transition on the optical resonances of this system through temperature-dependent variable angle extinction and photoluminescence measurements. Numerical simulations based on the finite element method are used to elucidate the tuning mechanism. As we show, from the interplay between coupling, detuning, and radiative losses of this system, a powerful approach emerges to tailor the emission spectrum and directionality of extended sources.

Figure 1 shows a sketch of the sample. First, CdSe/CdS/ZnS core-shell QDs were synthesized starting from CdSe seeds

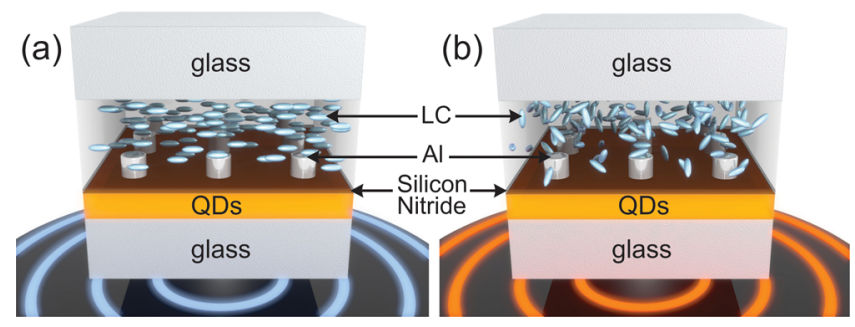

Figure 1. Schematic representation of the sample. (a) At roomtemperature $\left(\sim 23{ }^{\circ} \mathrm{C}\right)$ the liquid crystal is ordered, making the medium overlying the plasmonic antennas birefringent. (b) At higher temperatures $\left(>58{ }^{\circ} \mathrm{C}\right)$ the liquid crystal is disordered, yielding an isotropic refractive index in the same medium.

with a zinc blende structure ${ }^{44}$ through a successive ion layer adsorption and reaction procedure (SILAR). ${ }^{45}$ The resulting QDs had an average diameter of $6.5 \mathrm{~nm}$ and an emission peak at $585 \mathrm{~nm}$ (see Supporting Information for details on the synthesis and characterization of the QDs). The QDs, dispersed in toluene, were spin-coated on a glass substrate. This resulted in a $120 \mathrm{~nm}$ thick QD layer as determined by atomic force microscopy. A protective silicon nitride layer of $15 \mathrm{~nm}$ was deposited on top of the QD layer by plasma-enhanced chemical vapor deposition in order to planarize the surface. A relatively low temperature $\left(120^{\circ} \mathrm{C}\right)$ was used in the deposition to avoid degradation of the QDs (see Supporting Information for characterization of the $\mathrm{QD} /$ slicon nitride layer). Aluminum nanodisk arrays were fabricated on top of the silicon nitride layer by substrate conformal imprint lithography. ${ }^{46}$ The nanodisks have a nominal height of $150 \pm 20 \mathrm{~nm}$ and diameter of $120 \pm 20 \mathrm{~nm}$ and are arranged in a square lattice with a periodicity of $390 \pm 15 \mathrm{~nm}$. To control the LC orientation, we placed a thin layer of nylon alignment material over the array (not shown in Figure 1). This material is mechanically rubbed to force the LC to orient in a planar direction along one of the lattice vectors. Finally, UV curable glue containing spacer balls with a diameter of $6 \mu \mathrm{m}$ (not shown in Figure 1) is placed at the edge of the substrate, away from the array, to attach a glass plate to the sample. The space between the array and the top glass plate is filled with LC E7 (Merck) which has a critical temperature $T_{\mathrm{c}}=58{ }^{\circ} \mathrm{C}$. ${ }^{47}$

Figure 2a shows the normal incidence extinction spectrum of the sample for different temperatures. A collimated beam
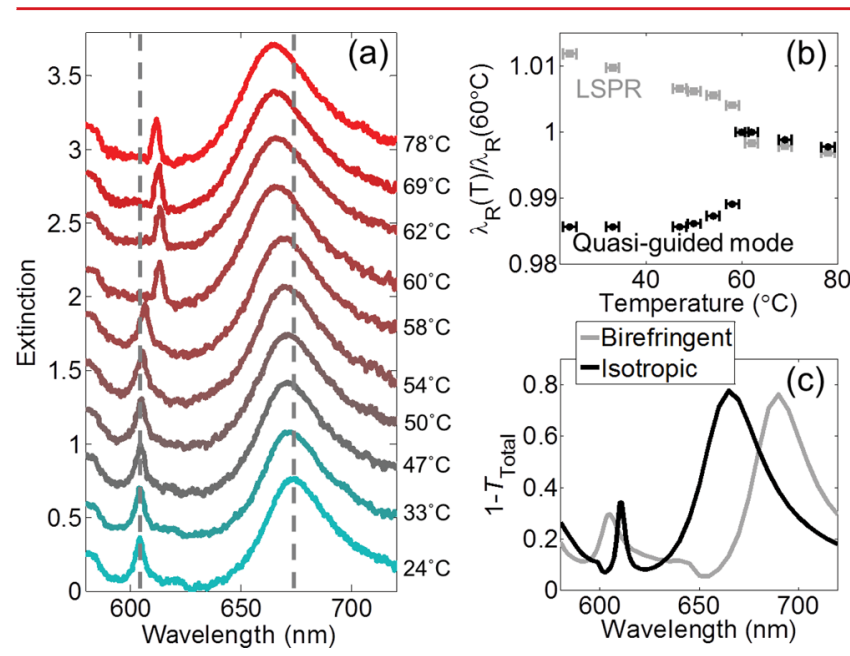

Figure 2. (a) Temperature-dependent normal incidence extinction measurements. The extinction is consecutively offset by 0.32 for clarity. The dashed gray lines are guides to the eye. (b) Temperaturedependent peak resonance wavelength normalized to the corresponding wavelength at $60 \pm 2{ }^{\circ} \mathrm{C}$ (criticality). (c) Simulated $1-T_{\text {Total }}$ spectra for birefringent and isotropic liquid crystal conditions, in correspondence to experiments at 24 and $60{ }^{\circ} \mathrm{C}$, respectively.

(angular spread $<0.1^{\circ}$ ) from a halogen lamp illuminates the sample with a linear polarization along the extraordinary index direction of the LC, which is aligned with one of the lattice vectors of the array. A fiber-coupled spectrometer measures the zeroth order transmittance $T_{0}$ in the far-field. The extinction follows as $1-T_{0}$. At all temperatures, the two peaks in extinction correspond to hybridized plasmonic-photonic resonances. These resonances arise from the coupling of LSPRs in the nanodisks to the fundamental transverse magnetic $\left(\mathrm{TM}_{0}\right)$ waveguide mode in the $\mathrm{QD}$ layer. Previous work has shown that the LSPR-waveguide mode mixing leads to hybrid modes known as waveguide-plasmon-polaritons. ${ }^{34,35}$ When the bare LSPR and waveguide mode wavelength detuning is zero, the emergent waveguide-plasmon-polaritons are half plasmon-like and half waveguide mode-like. In contrast, the LSPR and waveguide mode in our experiments are detuned (their peak wavelengths are significantly apart), but still 
coupled. The large detuning makes the coupled modes resemble one or the other of the bare modes. In Figure 2a particularly, the broad resonance at long wavelengths is reminiscent of the LSPR, while the sharper resonance at short wavelengths is reminiscent of a quasi-guided mode. The mode is quasi-guided because its radiative coupling to the antennas makes it leaky. In view of these effects, we shall hereafter refer to the broad resonance as the hybridized LSPR and to the narrow resonance as the quasi-guided mode, reserving the term waveguide-plasmon-polariton for the case where these hybrid modes are tuned in resonance. ${ }^{34,35}$

An interesting feature in Figure $2 \mathrm{a}$ is that the two resonances shift in opposite directions below $60{ }^{\circ} \mathrm{C}$, but in the same direction above $60^{\circ} \mathrm{C}$. To illustrate this effect, we plot in Figure $2 \mathrm{~b}$ the temperature-dependent peak wavelength of each resonance normalized to its peak wavelength at $60{ }^{\circ} \mathrm{C}$. The observed behavior for the quasi-guided mode and the LSPR is in qualitative agreement with the temperature-dependence of the ordinary index $n_{\mathrm{o}}$ and the extraordinary index $n_{\mathrm{e}}$, respectively, of the LC we use. ${ }^{47}$ Furthermore, the experimental value we retrieve for the critical temperature, $60 \pm 2{ }^{\circ} \mathrm{C}$, coincides within the error bar with the value reported by $\mathrm{Li}$ et al. $^{47}$ The error stems from spatial and temporal variations in temperature across the sample, which we characterized with an infrared camera to obtain a faithful map of the local temperature. All of the above observations strongly suggest that in the birefringent state (below $60{ }^{\circ} \mathrm{C}$ ), the hybridized LSPR mainly samples $n_{\mathrm{e}}$ while the quasi-guided mode mainly samples $n_{\mathrm{o}}$. The shifts in the extinction peaks are then due to a transition of the LC from an ordered (Figure 1a) to a disordered (Figure 1b) state, whereby the birefringence is removed and an isotropic refractive index sets in at high temperature. For the quasi-guided mode, the peak wavelength shift between 24 and $60{ }^{\circ} \mathrm{C}$ is $9 \mathrm{~nm}$, which is 3 times the line width at $60{ }^{\circ} \mathrm{C}$. An analysis of the peak shift normalized to the line width is provided in Figure S8 of the Supporting Information. The observed spectral shifts were experimentally verified to be reversible upon successive heating and cooling of the sample.

To elucidate the temperature dependence of the optical resonances, we simulated the response of the system using a finite element method (COMSOL). In the simulations, plane waves impinge from the LC layer, which is assumed to have infinite thickness above the array. Below $T_{\mathcal{O}}$ we assume that the LC layer is perfectly ordered and aligned along the rubbing direction. Consequently, the LC constitutes a homogeneous anisotropic material with a weakly dispersive ordinary $\left(n_{0} \approx\right.$ $1.52)$ and extraordinary $\left(n_{\mathrm{e}} \approx 1.73\right)$ refractive index. Above $T_{\mathrm{c}}$ the LC layer is assumed to be isotropic, with a refractive index $n_{\mathrm{c}}$ approximately given by the weighted average of $n_{\mathrm{o}}$ and $n_{\mathrm{e}}: n_{\mathrm{c}}$ $\approx\left(\left(2 n_{\mathrm{o}}+n_{\mathrm{e}}\right) / 3\right) \approx 1.59$. The frequency-dependent LC refractive index is taken from literature, ${ }^{47,48}$ for the QD layer and aluminum we have obtained them from ellipsometric measurements (see the Supporting Information, Figure S7), while the glass substrate is essentially dispersionless and lossless over the measurement range.

Figure $2 \mathrm{c}$ shows the $1-T_{\text {total }}$ spectra where $T_{\text {total }}$ is the simulated total transmittance. The peak resonance wavelengths and shift directions in the simulations are in good agreement with the experiments. To achieve this agreement, we simulated particles with a height and diameter of $100 \mathrm{~nm}$, arranged in a lattice with constant $a=378 \mathrm{~nm}$. These dimensions are slightly different from the nominal values of the fabricated structures, possibly due to deviations in the fabrication process. In addition, small discrepancies between the simulated and experimental refractive indices could also exert an influence on our results.

The connection between the sharp resonance at shorter wavelengths in our measurements with a waveguide mode in the QD layer was established through eigenmode simulations using COMSOL. We calculated the dispersion of the fundamental TM waveguide mode in the same multilayer structure discussed above, but without the nanodisk array. Both anisotropic and isotropic LC conditions were considered. The simulations indicate that the multilayer structure supports an eigenmode-the fundamental transverse magnetic (TM) waveguide mode in the QD layer-with a dispersion relation closely following the dispersion relation of the sharp feature in our measurements. The dispersion of this mode is shown below, in Figure 5, in connection to angle-resolved data. While such a guided mode is bound to the high refractive index layer in absence of the nanodisk array, the periodic array can couple a normal incident plane wave into (or out of) this mode at wavelengths close to the diffraction edge. This is the origin of the sharp resonance in the measurements of Figure 2a, which is related to the plane wave excitation of a guided mode via the first diffraction order. We furthermore verified through full field simulations that when the QD layer thickness is increased, the short wavelength resonance red-shifts (see the Supporting Information, Figure S4). This is in agreement with the expected behavior of the fundamental waveguide mode in the QD layer.

To examine the coupled nature of the modes in more detail, we plot the field enhancement $|\mathbf{E}| /\left|\mathbf{E}_{\text {input }}\right|$ at an $x z$-plane intersecting the nanodisks at their center. We do this for the hybridized LSPR in Figure 3a and for the quasi-guided mode in

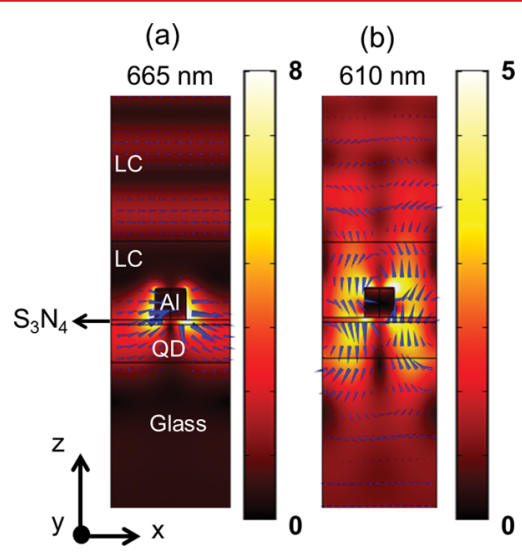

Figure 3. Total field enhancement for isotropic LC (above $T_{\mathrm{c}}$ ) at a wavelength of (a) $665 \mathrm{~nm}$ (hybridized LSPR) and (b) $610 \mathrm{~nm}$ (quasiguided mode). The incoming field is polarized along $x$ (along the LC extraordinary index direction). For all graphs, the blue arrows show the $E$ field at a certain phase.

Figure $3 b$, both in the isotropic state. The field profiles are very similar in the birefringent state. In Figure $3 a$ the field enhancement is localized near the metallic nanostructure, resembling the characteristics of bare LSPRs. In contrast, Figure $3 \mathrm{~b}$ shows delocalized field enhancements characteristic of a waveguide mode close to cutoff, as it may be expected due to the small waveguide thickness and the asymmetry of the refractive index of the upper and lower media. Note, however, that this delocalized field enhancement is relatively strong in the vicinity of the nanodisks, indicating once more that 
waveguide-LSPR coupling is present. It should be mentioned that all of these properties of the modes are a sensitive function of the structural parameters and material composition. For example, for an increased lattice constant the waveguide mode dispersion will red-shift, while for an increased diameter of the nanodisks the LSPR will red-shift and broaden. In addition, the height of the nanodisks also influences the field extension of the modes into the LC, thus affecting their sensitivity to the LC phase transition. To give an indication of the role that the metallic nanoantennas play in the effects here reported, we provide in Figure S6 of the Supporting Information simulation results for a lattice of silicon nitride particles in otherwise identical conditions. As shown therein, for the dielectric nanoparticles the peak near the quasi-guided mode resonance is strongly suppressed in the birefringent state. We attribute this to the reduced polarizability of the dielectric nanoparticles, which gives a weaker scattering power into (or out of) the guided mode. While this is far from an exhaustive study, our results indicate that metallic nanoantennas can lead to different effects than dielectric ones.

An interesting observation in Figure 3 concerns the dominant field components of each mode. In fact, this is the reason behind the different temperature-dependent shift directions shown in Figure 2. The hybridized LSPR has a dominant electric field component parallel to the input plane wave polarization, as shown by the blue arrows in Figure $3 \mathrm{a}$. This is parallel to $n_{\mathrm{e}}$. In contrast, the quasi-guided mode has a dominant electric field component along the $z$-direction, as shown by the blue arrows in Figure $3 \mathrm{~b}$. This is parallel to $n_{\mathrm{o}}$. Note that the relation between the LC refractive indices is $n_{\mathrm{o}}<$ $n_{\mathrm{c}}<n_{\mathrm{e}}$. Therefore, in the transition toward the isotropic state, the quasi-guided mode experiences an increase of effective index (and therefore a red-shift), while the opposite occurs for the hybridized LSPR (it blue-shifts).

In the following, we present temperature-dependent measurements of photoluminescence enhancement (PLE) by the metallic nanoantennas. The sample was pumped by a 450 $\mathrm{nm}$ laser beam at a fixed angle of incidence $\left(5^{\circ}\right)$ from the normal. The light emitted at different directions was collected by the same fiber-coupled spectrometer used for the extinction measurements. The PLE is defined as $I_{\text {in }} / I_{\text {out }}$ with $I_{\text {in }}$ the emitted intensity from the QDs in the presence of the nanodisk array, and $I_{\text {out }}$ without the array. Figure 4a shows the PLE spectra in the direction normal to the periodicity plane, at the same temperatures as in Figure 2a. The wavelength range is limited by the emission bandwidth of the QDs. Figure $4 \mathrm{~b}$ shows the temperature-dependent wavelength shift of the quasiguided mode normalized to the line width. Both the shift and the line width are referenced to the measurements at $60^{\circ} \mathrm{C}$. As in extinction, the quasi-guided mode peak first red-shifts with increasing temperature up to $60{ }^{\circ} \mathrm{C}$ and then blue-shifts for higher temperatures. At $60{ }^{\circ} \mathrm{C}$ and above, the line width is slightly narrower, and the magnitude of the PLE is slightly greater than below $60{ }^{\circ} \mathrm{C}$. By fitting the PLE peak at $60{ }^{\circ} \mathrm{C}$ with the Fano-shape function we found a line width of $5.5 \mathrm{~nm}$, which is remarkably narrow for plasmonic-coupled systems. The Fano shape was used for the fitting as that is the expected spectral shape of modes arising from the coupling between a narrow (waveguide) resonance with a broad (LSPR) resonance. ${ }^{49,50}$ These results demonstrate the strength of hybrid plasmonicphotonic modes for emission enhancement purposes by providing a strong near field enhancement while having a low loss. We highlight that maximizing the PLE factor is not the
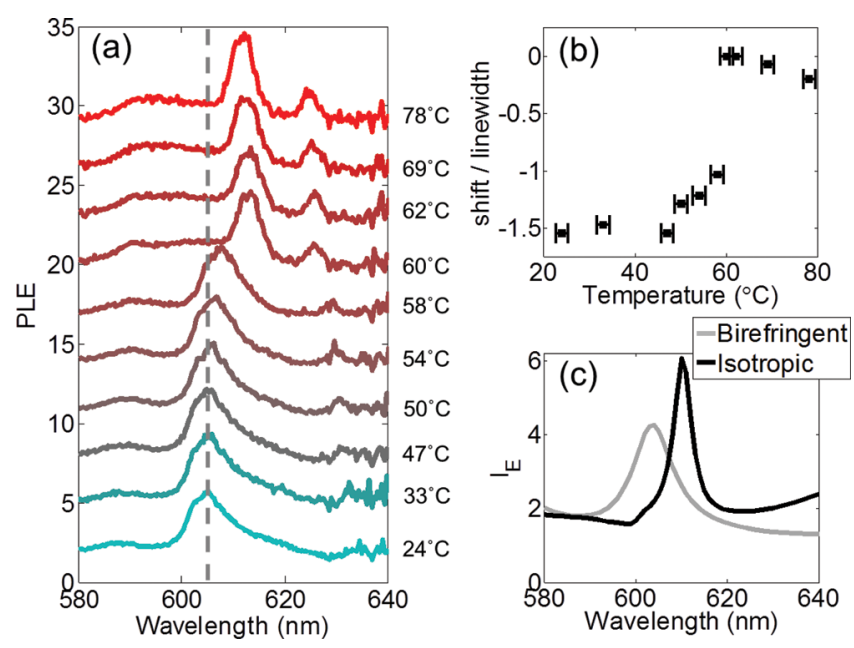

Figure 4. (a) Measured temperature-dependent photoluminescence enhancement (PLE) in the direction normal to the sample. The PLE is consecutively offset by 3 for clarity. The dashed gray line is a guide to the eye. (b) Measured peak wavelength shift $\left(D_{\lambda}\right)$ of the quasi-guided mode PLE peak normalized to its line width at $60{ }^{\circ} \mathrm{C}$. Both the peak wavelength and the line width are referenced to the measurement at $60{ }^{\circ} \mathrm{C}$. (b) Simulated electric field intensity enhancement integrated over the QD layer, $I_{E}$, in the birefringent and isotropic LC state.

subject of our study, as this has been done in previous works. ${ }^{41}$ Instead, we demonstrate the possibility to actively control the PLE by means of an external tuning parameter (temperature in this case).

To elucidate the PLE measurements, we simulate the spectral dependence of the field enhancement in the QD layer. We define the electric field intensity enhancement in the QD layer by the nanodisk array with respect to the bare layer (without the array) as $I_{E}=\left(\left(\int|E|_{\text {with array }}^{2} \mathrm{~d} V\right) /\left(\int|E|_{\text {without array }}^{2} \mathrm{~d} V\right)\right)$, where the volume integral is done over the $\mathrm{QD}$ layer. While in the simulations $I_{E}$ is a measure of the excitation strength of an optical mode, by reciprocity it also represents a decay strength of the same mode to outgoing plane waves with the same direction. Thus, $I_{E}$ correlates with the PLE in the measurements. Figure $4 \mathrm{c}$ shows $I_{E}$ for a plane wave at normal incidence. The simulations are in good agreement with PLE measurements, both displaying a red-shift and line width narrowing when going from the birefringent state to the isotropic state. Furthermore, the peak PLE also increases. The line width narrowing is associated with a slightly weaker confinement of the mode to the metallic structure in the isotropic state, as shown in Figure S5 of the Supporting Information.

We now analyze the directional dependence of the extinction and PLE spectra. Figure 5a,b shows the measured extinction at 24 and $60{ }^{\circ} \mathrm{C}$, respectively, while Figure 5c,d displays the PLE at the same two temperatures. In all plots the bands of enhanced extinction or PLE correspond to the excitation of quasi-guided modes. The variable angle data show a similar shift of this resonance in both extinction and PLE over the entire band when the LC layer transitions from birefringent to isotropic, as observed at normal incidence (Figure 2a and Figure 4a). Consequently, the LC transition enables us to actively tune the directionality of the emission at a given wavelength. In Figure S11 of the Supporting Information, we provide an analysis of the temperature-induced peak angle shift at $605 \mathrm{~nm}$. Due to the dispersion of the band and an exceptionally low angular spread $\left(\leq 1.5^{\circ}\right)$, we demonstrate that 
(a)

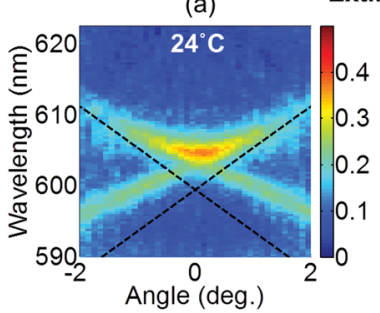

(c)

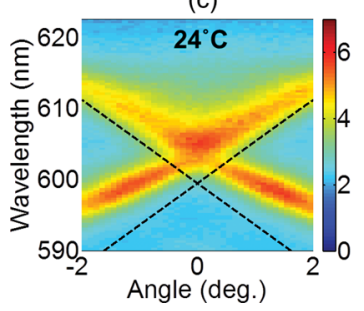

Extinction

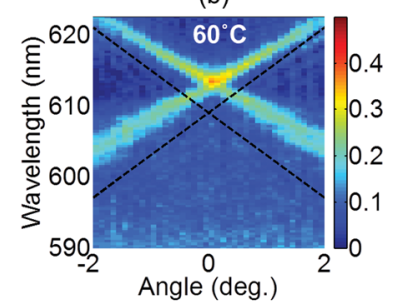

PLE

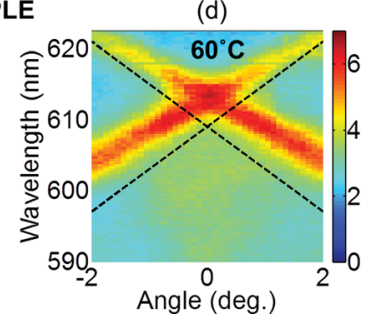

Figure 5. Measured variable angle extinction spectra at (a) $24{ }^{\circ} \mathrm{C}$ and (b) $60{ }^{\circ} \mathrm{C}$. Measured variable angle photoluminescence enhancement spectra at (c) $24{ }^{\circ} \mathrm{C}$ and (d) $60{ }^{\circ} \mathrm{C}$. The black dashed lines in all the plots represent the dispersion of the fundamental TM waveguide mode in the absence of the aluminum nanodisk array.

the shift is greater than the angular spread. The change in directionality at a given wavelength is not associated with a reduced emitted power. As shown in Figure S11 of the Supporting Information, at $60{ }^{\circ} \mathrm{C}$ there are two emission lobes, and the power in each lobe is similar to the power in the direction perpendicular to the periodicity plane at $23{ }^{\circ} \mathrm{C}$.

Comparing Figure $5 \mathrm{a}$ with Figure $5 \mathrm{c}$, and Figure $5 \mathrm{~b}$ with Figure $5 \mathrm{~d}$, one sees that the dispersion of the quasi-guided mode in PLE closely resembles that in extinction. Furthermore, in all dispersion diagrams the quasi-guided mode closely follows the calculated dispersion of the fundamental TM waveguide mode for the structure without the nanodisk array, indicated by the dashed lines in Figure 5. The dispersion of this mode has been folded into the first Brillouin zone of the nanoantenna array. The proximity in wavelength of the quasi-guided mode to the bare waveguide mode suggests once more that the hybrid mode resembles the bare mode, and that the coupling to the LSPR has a small influence due to the large detuning between the modes. This is, however, not a detriment for our purpose of actively tuning the PLE due to the quasi-guided mode via the LC phase transition. In fact, the large out-of-plane fields of the bare TM polarized guided mode (and also of the coupled mode resembling the bare one) make it more sensitive to refractive index changes in the LC layer. Clearly, this allows for a greater degree of tunability given the fixed contrast between $n_{\mathrm{e}}$ and $n_{\mathrm{o}}$ of the LC.

In conclusion, we have demonstrated active spectral and directional control of the enhanced light emission of quantum dots coupled to metallic nanoantennas. For this purpose, we employed a hybrid plasmonic-photonic resonance emerging from the radiative coupling between localized surface plasmons and a waveguide mode in a quantum dot emitter layer. The tuning was achieved by covering the array with a thermotropic liquid crystal, which changes from a birefringent to an isotropic state above the critical temperature. In turn, this modifies the resonance conditions of the coupled system. The narrow line width $(<6 \mathrm{~nm})$ and angular spread $\left(<1.5^{\circ}\right)$ of this resonance allowed us to tune the emission with unprecedented wavelength and angular specificity. Supporting our experiments with numerical simulations, we have shown that the tuning mechanism depends on the field profile and more specifically on the orientation of the dominant mode polarization in the LC layer with respect to the LC axis. Having here provided a proof-of-principle experiment, we envisage future studies to optimize both structural and material parameters in order to achieve even stronger effects. For instance, with greater field overlaps (coupling strengths), this system could enter into the strong coupling regime, where the waveguide-plasmon coupling rate exceeds the loss rates. In this case, active control of strong coupling appears as a fundamentally interesting possibility. ${ }^{51}$ From an applied perspective, we believe that these results hold great promise for smart lighting applications where active beaming and color tuning is required. This is especially useful in the wavelength range where we work, where the human eye translates small variations in wavelength to relatively large color changes. For practical implementations, it could be interesting to achieve active tuning of nanoantenna-enhanced light emission by electrical means, i.e., by applying a voltage. We do not expect major changes in behavior for electrical tuning because the wavelength shift is not a consequence of the tuning mechanism, but rather of the refractive index contrast of the LC in the birefringent state.

\section{ASSOCIATED CONTENT}

\section{Supporting Information}

Details on the synthesis and characterization of the quantum dots, characterization of the silicon nitride layer, numerical simulations of the waveguide mode as a function of the quantum dot layer thickness, and the field profiles of the quasiguided mode above the liquid crystal critical temperature. This material is available free of charge via the Internet at http:// pubs.acs.org.

\section{AUTHOR INFORMATION}

\section{Corresponding Author}

*E-mail: bjorn.maes@umons.ac.be.

\section{Author Contributions}

A.A. and S.R.-K.R. had an equal contribution.

\section{Notes}

The authors declare no competing financial interest.

\section{ACKNOWLEDGMENTS}

This work was supported by the Interuniversity Attraction Poles program of the Belgian Science Policy Office under Grant No. IAP P7-35 photonics@be, The Netherlands Foundation for Fundamental Research on Matter (FOM), and The Netherlands Organization for Scientific Research (NWO) and is part of an industrial partnership program between Philips and FOM.

\section{REFERENCES}

(1) Bharadwaj, P.; Deutsch, B.; Novotny, L. Adv. Opt. Photon. 2009, $1,438-483$.

(2) Curto, A. G.; Volpe, G.; Taminiau, T. H.; Kreuzer, M. P.; Quidant, R.; van Hulst, N. F. Science 2010, 329, 930-933.

(3) Livneh, N.; Strauss, A.; Schwarz, I.; Rosenberg, I.; Zimran, A.; Yochelis, S.; Chen, G.; Banin, U.; Paltiel, Y.; Rapaport, R. Nano Lett. 2011, 11, 1630-1635.

(4) Coenen, T.; Vesseur, E. J. R.; Polman, A.; Koenderink, A. F. Nano Lett. 2011, 11, 3779-3784.

(5) Belacel, C.; Habert, B.; Bigourdan, F.; Marquier, F.; Hugonin, J.P.; Michaelis de Vasconcellos, S.; Lafosse, X.; Coolen, L.; Schwob, C.; 
Javaux, C.; Dubertret, B.; Greffet, J.-J.; Senellart, P.; Maitre, A. Nano Lett. 2013, 13, 1516-1521.

(6) Hancu, I. M.; Curto, A. G.; Castro-López, M.; Kuttge, M.; van Hulst, N. F. Nano Lett. 2014, 14, 166-171.

(7) Mertens, H.; Biteen, J. S.; Atwater, H. A.; Polman, A. Nano Lett. 2006, 6, 2622-2625.

(8) Ming, T.; Zhao, L.; Yang, Z.; Chen, H.; Sun, L.; Wang, J.; Yan, C. Nano Lett. 2009, 9, 3896-3903.

(9) Anger, P.; Bharadwaj, P.; Novotny, L. Phys. Rev. Lett. 2006, 96, 113002.

(10) Kühn, S.; Håkanson, U.; Rogobete, L.; Sandoghdar, V. Phys. Rev. Lett. 2006, 97, 017402.

(11) Muskens, O. L.; Giannini, V.; Sánchez-Gil, J. A.; Gomez Rivas, J. Nano Lett. 2007, 7, 2871-2875.

(12) Ringler, M.; Schwemer, A.; Wunderlich, M.; Nichtl, A.; Kürzinger, K.; Klar, T. A.; Feldmann, J. Phys. Rev. Lett. 2008, 100, 203002.

(13) Jin, P.; Tazawa, M.; Xu, G. J. Appl. Phys. 2006, 99, 096106.

(14) Olcum, S.; Kocabas, A.; Ertas, G.; Atalar, A.; Aydinli, A. Opt. Express 2009, 17, 8542-8547.

(15) Jun, Y. C.; Huang, K. C. Y.; Brongersma, M. L. Nat. Commun. 2011, 2, 283, 10.1038/ncomms1286.

(16) Beeckman, J.; Neyts, K.; Vanbrabant, P. J. M. Opt. Eng. 2011, 50, 081202-081202-17.

(17) Shadrivov, I. V.; Kapitanova, P. V.; Maslovski, S. I.; Kivshar, Y. S. Phys. Rev. Lett. 2012, 109, 083902.

(18) Lumdee, C.; Toroghi, S.; Kik, P. G. ACS Nano 2012, 6, 63016307

(19) Müller, J.; Sönnichsen, C.; von Poschinger, H.; von Plessen, G.; Klar, T. A.; Feldmann, J. Appl. Phys. Lett. 2002, 81, 171-173.

(20) Kossyrev, P. A.; Yin, A.; Cloutier, S. G.; Cardimona, D. A.; Huang, D.; Alsing, P. M.; Xu, J. M. Nano Lett. 2005, 5, 1978-1981.

(21) Chu, K. C.; Chao, C. Y.; Chen, Y. F.; Wu, Y. C.; Chen, C. C. Appl. Phys. Lett. 2006, 89, 103107.

(22) Evans, P. R.; Wurtz, G. A.; Hendren, W. R.; Atkinson, R.; Dickson, W.; Zayats, A. V.; Pollard, R. J. Appl. Phys. Lett. 2007, 91, 043101.

(23) Dickson, W.; Wurtz, G. A.; Evans, P. R.; Pollard, R. J.; Zayats, A. V. Nano Lett. 2008, 8, 281-286.

(24) Xiao, S.; Chettiar, U. K.; Kildishev, A. V.; Drachev, V.; Khoo, I. C.; Shalaev, V. M. Appl. Phys. Lett. 2009, 95, 033115.

(25) Dridi, M.; Vial, A. J. Phys. Chem. C 2010, 114, 9541-9545.

(26) Khatua, S.; Chang, W.-S.; Swanglap, P.; Olson, J.; Link, S. Nano Lett. 2011, 11, 3797-3802.

(27) Li, J.; Ma, Y.; Gu, Y.; Khoo, I.-C.; Gong, Q. Appl. Phys. Lett. 2011, 98, 213101.

(28) Li, H.; Xu, S.; Gu, Y.; Wang, K.; Xu, W. Appl. Phys. Lett. 2013, $102,051107$.

(29) Maier, S. A. Plasmonics: Fundamentals and Applications; Springer: New York, 2007; pp 607-636.

(30) Wokaun, A.; Lutz, H.-P.; King, A. P.; Wild, U. P.; Ernst, R. R. J. Chem. Phys. 1983, 79, 509.

(31) Zou, S.; Schatz, G. C. J. Chem. Phys. 2004, 121, 12606-12612.

(32) Hicks, E. M.; Zou, S.; Schatz, G. C.; Spears, K. G.; Van Duyne, R. P.; Gunnarsson, L.; Rindzevicius, T.; Kasemo, B.; Käll, M. Nano Lett. 2005, 5, 1065-1070.

(33) García de Abajo, F. J.; Gómez-Medina, R.; Sáenz, J. J. Phys. Rev. E 2005, 72, 016608 .

(34) Christ, A.; Tikhodeev, S. G.; Gippius, N. A.; Kuhl, J.; Giessen, H. Phys. Rev. Lett. 2003, 91, 183901.

(35) Rodriguez, S. R. K.; Murai, S.; Verschuuren, M. A.; Rivas, J. G. Phys. Rev. Lett. 2012, 109, 166803.

(36) Teperik, T. V.; Degiron, A. Phys. Rev. B 2012, 86, 245425.

(37) Abass, A.; Rodriguez, S. R.-K.; Gómez Rivas, J.; Maes, B. ACS Photonics 2014, 1, 61-68.

(38) Vecchi, G.; Giannini, V.; Gómez Rivas, J. Phys. Rev. Lett. 2009, $102,146807$.

(39) Pellegrini, G.; Mattei, G.; Mazzoldi, P. J. Phys. Chem. C 2011, $115,24662-24665$.
(40) Rodriguez, S. R. K.; Lozano, G.; Verschuuren, M. A.; Gomes, R.; Lambert, K.; Geyter, B. D.; Hassinen, A.; Thourhout, D. V.; Hens, Z.; Rivas, J. G. Appl. Phys. Lett. 2012, 100, 111103.

(41) Lozano, G.; Louwers, D. J.; Rodriguez, S. R. K.; Murai, S.; Jansen, O. T.; Verschuuren, M. A.; Gomez Rivas, J. Light Sci. Appl. 2013, 2, e66.

(42) Zhou, W.; Dridi, M.; Suh, J. Y.; Kim, C. H.; Co, D. T.; Wasielewski, M. R.; Schatz, G. C.; Odom, T. W. Nat. Nanotechnol. 2013, 8, 506-511.

(43) Hu, C.; Aubert, T.; Justo, Y.; Flamee, S.; Cirillo, M.; Gassenq, A.; Drobchak, O.; Beunis, F.; Roelkens, G.; Hens, Z. Nanotechnology 2014, 25, 175302.

(44) Flamee, S.; Cirillo, M.; Abe, S.; De Nolf, K.; Gomes, R.; Aubert, T.; Hens, Z. Chem. Mater. 2013, 25, 2476-2483.

(45) Li, J. J.; Wang, Y. A.; Guo, W.; Keay, J. C.; Mishima, T. D.; Johnson, M. B.; Peng, X. J. Am. Chem. Soc. 2003, 125, 12567-12575.

(46) Verschuuren, M. A. Substrate Conformal Imprint Lithography for Nanophotonics. Ph.D. Dissertation, Utrecht University, 2010.

(47) Li, J.; Wu, S.-T.; Brugioni, S.; Meucci, R; Faetti, S. J. Appl. Phys. 2005, 97, 073501.

(48) Li, J.; Wen, C.-H.; Gauza, S.; Lu, R.; Wu, S.-T. J. Display Technol. 2005, 1,51 .

(49) Luk'yanchuk, B.; Zheludev, N. I.; Maier, S. A.; Halas, N. J.; Nordlander, P.; Giessen, H.; Chong, C. T. Nat. Mater. 2010, 9, 707715 .

(50) Miroshnichenko, A. E.; Flach, S.; Kivshar, Y. S. Rev. Mod. Phys. 2010, 82, 2257-2298.

(51) Schwartz, T.; Hutchison, J. A.; Genet, C.; Ebbesen, T. W. Phys. Rev. Lett. 2011, 106, 196405. 